\title{
Research on Calculation Method Based on Information Entropy about Traffic Signs in China
}

\author{
Yaping Zhang ${ }^{1}$, Shouming $\mathbf{Q i}^{1}$, Yanli Ma ${ }^{1}$, Liwei $\mathrm{Hu}^{2}$ \\ ${ }^{1}$ School of Transportation Science and Engineering, Harbin Institute of Technology \\ Huanghe Road, No.73, Nangang District, Harbin, PR.China \\ zxlt0905@163.com; shouming1991@163.com; mayanlihit@163.com \\ ${ }^{2}$ Faculty of Transportation Engineering, Kunming University of Science and Technology \\ Jingming South road, No.727, Chenggong District, Kunming, China \\ liweihukm@sina.com
}

\section{Extended Abstract}

Driving on the highway, drivers can make accurate operation only by making effective perception and judgment of the obtained effective information transferred from highway traffic engineering facilities. Therefore, it is required that not only should we make reasonable designs and setting for the traffic engineering facilities, and a miscellaneous design and set for some important traffic information, we should also ensure that the drivers have a good dynamic visibility and can cope with the environment by a sufficient amount of information transferred from highway traffic engineering facilities. A lot of useful researches and studies have carried out in the relevant aspects of the traffic information quantity at home and abroad, and they got a lot of achievements. But those researches primarily focused on the macroscopic qualitative analysis or aimed at a single category of traffic engineering facilities of traffic information threshold, which lack of systematic overall discussion for highway engineering facilities information quantity or model construction.

Based on analysis of the relevant research domestic and overseas, this paper uses the quantitative argumentation mainly and the qualitative analysis as a supplement. It puts forward some concepts about information quantity transferred from highway traffic engineering facilities, mutual information and information entropy. According to the basic elements of traffic engineering facilities, it also builds a basic amount of information computational model which serves for the highway traffic engineering facilities from the angle of the eight types of information (Chinese character information, English character information, Arabic numerals information, Geometric shapes information ,Colour information ,Guidance information, Graph or characteristic symbol information and linear facilities information ,et al .Minority characters is not considered temporarily here.). Besides, to analyse the theoretical information content which can be obtained by drivers in the unit road space, this paper proposes a concept called transmission information density of highway traffic engineering facilities and does a case analysis for the model. Taking Hada highway as an example (Harbin-Zhaodong section, about $55 \mathrm{kms}$ ), we analysed the information quantity provided by this section of the highway traffic engineering facilities. Through calculating the total information quantity that Hada highway traffic facilities transfers, we receive the total information quantity which provided by the drivers transferred for highway traffic engineering facilities along the Hada highway from Harbin to Zhaodong, we learned its physical significance, namely Hada highway (from Harbin to Zhaodong direction) can provide an average of 85.15 bits per Kilometer of information quantity to the traffic engineering facilities along the highway.

This paper is intended to provide valuable references and experiences for the road traffic management department and the sector which formulate the traffic regulations and standards. It provides theoretical basis for designing and setting more reasonable traffic engineering facilities by quantitative analysis of the efficiency of the highway traffic engineering facilities. The research in this paper has a certain theoretical significance and practical value for improving the service capacity, operational efficiency and safety level of the highway traffic system. This research is supported by the National Key Research and Development Program of China (No. 2017YFC0803901); Scientific and Technological Research Program in Henan Province (No. 182102310765)

Keywords: Transportation Engineering, Traffic Facilities, Calculation Method, Information Entropy. 